\title{
Mittelfuß- und Zehenfrakturen
}

\author{
Dominik Heim, Ulrich Stricker
}

\section{Zusammenfassung}

Metatarsale- und Zehenfrakturen sind häufige Frakturen. Ihre Behandlung kann operativ oder konservativ sein - Ziel jeder Behandlung ist eine Wiederherstellung des anatomischen Fußgewölbes. Die Heilungsdauer beträgt mit/ ohne Osteosynthese rund 4 Wochen. An operativen Möglichkeiten bieten sich je nach Persönlichkeit der Fraktur Platten-/Schraubenosteosynthesen, Fixateur externe oder Kirschner-Draht-Fixationen an. $\mathrm{Zu}$ unterscheiden sind diese Frakturen von proximalen Metatarsale-V-frakturen, welche eine eigene Einheit darstellen und entsprechend der Einteilung nach Holzach [3] und Torg [8] konservativ oder operativ behandelt werden.

\section{Einleitung}

Vorfußfrakturen sind häufige Frakturen am menschlichen Skelett. Der häufigste Unfallmechanismus ist ein direktes Trauma durch Schlag oder Quetschung, eine repetitive Belastung bei Sportlern und Balletttänzern kann zu den typischen Stressfrakturen im Metatarsalebereich führen. Ziel jeder Behandlung ist die Wiederherstellung des Längs- und Quergewölbes des Vorfußes. Die Osteosynthese bietet - sofern indiziert - die Möglichkeit der frühfunktionellen Nachbehandlung mit dem Ziel posttraumatische Schwellungszustände und die Entwicklung einer Sudeck'schen Dystrophie zu verhindern [2].

Bei der Evaluation einer operativen Behandlung ist zu bedenken, dass im Stehen

OP-JOURNAL 2001; 17: 60-64

(c) Georg Thieme Verlag Stuttgart · New York die Hauptbelastung auf dem Metatarsale I, beim Laufen und Springen die Hauptbelastung auf dem Metatarsale II stattfindet. Deshalb sind diese zwei Knochen zusammen mit dem lateralen Randstrahl (Metatarsale $\mathrm{V}$ ) bei der Rekonstruktion im Vorfußbereich besonders wichtig [2].

Zur Beurteilung der Fraktursituation reichen häufig konventionelle Röntgenbilder des Fußes/Vorfußes in anteroposteriorem und schrägem Strahlengang.

Zusätzliche Abklärungen mittels Computertomographie empfehlen sich bei tarsometatarsalen Dislokationen und Gelenksfrakturen.

\section{Metatarsale-Frakturen (ausgenommen proximale Metatarsale V-Fraktur)}

Indikationen für eine operative Versorgung sind:

- dislozierte Schaftfrakturen

- dislozierte subkapitale Frakturen

- Gelenks- und periartikuläre Frakturen

Möglichkeiten der operativen Frakturfixation sind:

\section{Der Fixateur externe}

Indiziert vorwiegend bei Metatarsale-IFrakturen, bei denen die Weichteilsituation (Quetschung, höhergradig offene Fraktur) oder ein kurzes, gelenknahes Hauptfragment (gelenküberbrückender Fixateur externe) eine interne Fixation verunmöglichen. Bei gelenksnahen Frakturen erlaubt eine zusätzliche interne Minimalosteosynthese (perkutane Schrauben) eine frühzeitige Entfernung des äußeren, gelenküberbrückenden Gestänges (Abb.1). Bei Impressionsfrakturen an der Basis des Metatarsale (PilonTyp) kann eine Wiederherstellung der Gelenksfläche durch die Ligamentotaxis mittels einer Gelenksüberbrückung durch einen Fixateur, eventuell in Kombination mit einer Spongiosaplastik, erreicht werden (Abb.2). Im Allgemeinen eignet sich dazu ein kleiner AO fixateur externe in der unilateralen Konfiguration, eventuell in der Dreirohrkonstruktion.

\section{Platten- und Schraubenosteosynthese}

Dies dürfte die häufigst angewandte Methode darstellen [2]. Dabei kommen Implantate der Dimension 2,0-2,7 mm (Viertelrohrplatte) am Metatarsale II-V, am Metatarsale I der Dimension 3,5 mm (Drittelrohrplatte) zur Anwendung. Je nach Frakturtyp handelt es sich dabei um rigide Fixationen mit interfragementärer und/oder axialer Kompression bei einfachen Quer- oder Torsionsfrakturen (Abb.3) oder um frakturüberbrückende Fixationen bei mehrfragmentären, segmentalen Frakturen. Besonderes Augenmerk gilt dabei den distalen Metatarsale-V-Frakturen (subkapital mit diaphysärer Ausdehnung), welche geschlossen in ihrer Fehlstellung nicht korrigiert werden können und aufgrund ihrer exponierten Lage (lateraler Fußrand) bei konservativer Behandlung häufig in einer Pseudoarthrose enden. Hier kommen T-Platten der Dimension 2,7 mm oder kleine Kondylenplatten zur Anwendung.

\section{Kirschner-Draht-Osteosynthese}

In der Vergangenheit bei Metatarsaleschaftfrakturen der Strahlen II - IV häufig angewandt, da mit offener Reposition durch einen limitierten, dorsalen Zugang einfach durchzuführen (intramedulläre Markdrahtschienung), haben sie den Nachteil eines aus der Haut herausragenden Kirschner-Drahtes im plantaren Metatarsaleköpfchenbereich. Für die frühfunktionelle Nachbehandlung mit einer Abrollbelastung eignen sich heute die dorsalen Plattenosteosynthesen in der Dimension 2,0 mm (Minifragmentinstrumentarium) vorzüglich (Abb.4). Nachteil dieser Technik ist jedoch der extensivere Weichteilzugang und die Notwendigkeit der späteren Implantatentfernung. 

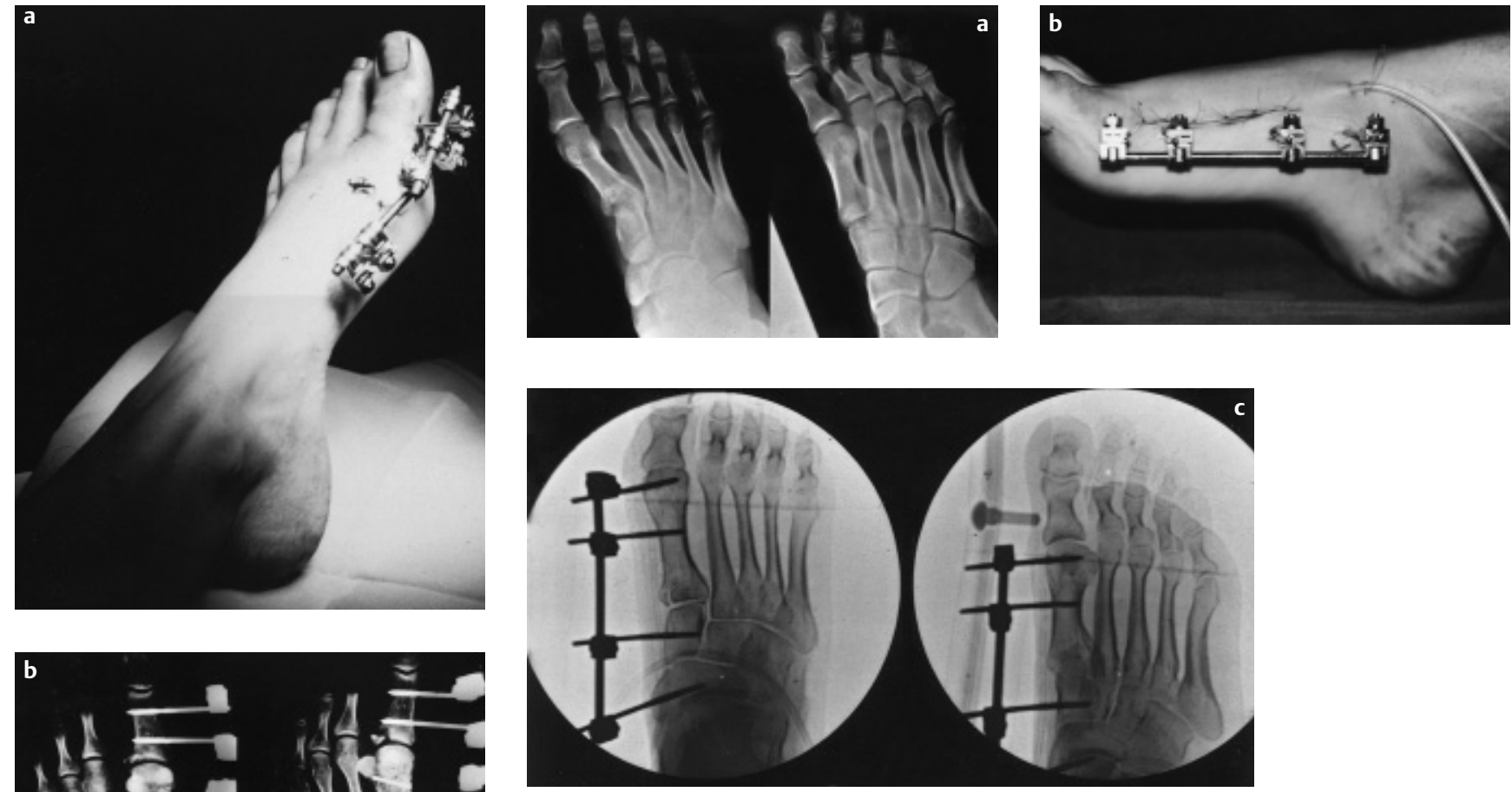

Abb. 2 Basisfraktur Metatarsale I mit Gelenksimpression durch Stauchungstrauma. Versorgung mit unilateralem, gelenküberbrückendem, kleinen AO Fixateur externe (1-Rohrkonstruktion) und Spongiosaplastik. (a) Unfallbild, (b) klinischer Zustand, (c) postoperatives Röntgenbild.

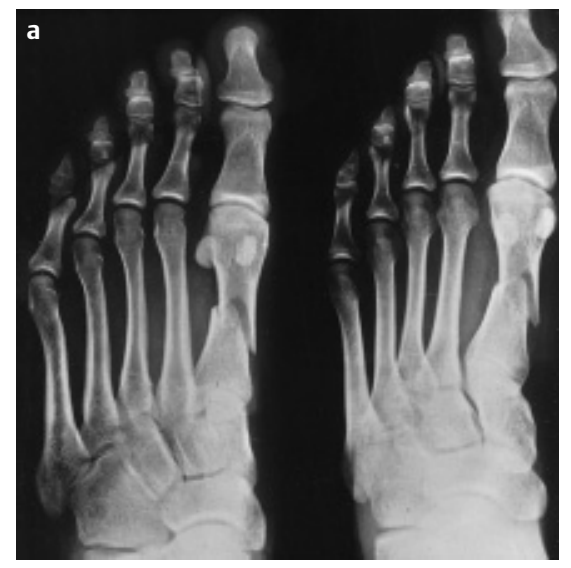

Abb. 3 Diaphysäre Schrägfraktur des Metatarsale I: Versorgt mit plattenunabhängiger Zugschraube und Drittelrohrplatte. (a) Unfallbild, (b) 1-Jahres-Röntgenbild.

\section{Proximale Frakturen des Metatarsale V}

Ganz anders geartet ist die Problematik der proximalen Frakturen des Metatarsale V. Bei den eigentlichen Basisfrakturen handelt es sich um die häufigste Fraktur des menschlichen Fußes. Fälschlicherweise wird sie häufig als Jones-Fraktur bezeichnet. Der Unfallmechanismus ist eine forcierte Adduktion des Fußes im Sinne einer Avulsionsfraktur. Topogra-

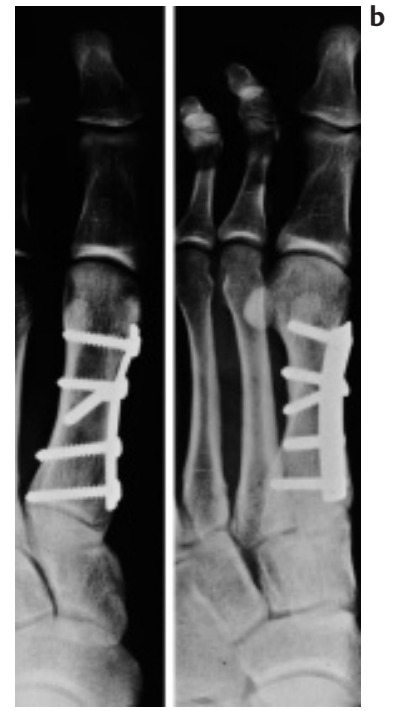

phisch gesehen setzen die Sehne des Peronaeus brevis, der abductor digiti minimi und der laterale Zügel der Plantaraponeurose an dieser Basis an. Durch repetitive Adduktionsbewegungen zum Beispiel bei chronischer Instabilität im oberen Sprunggelenk und bei Genu varum kommt es zu Stressfrakturen im proximalen Metatarsale-V-bereich [9]. 

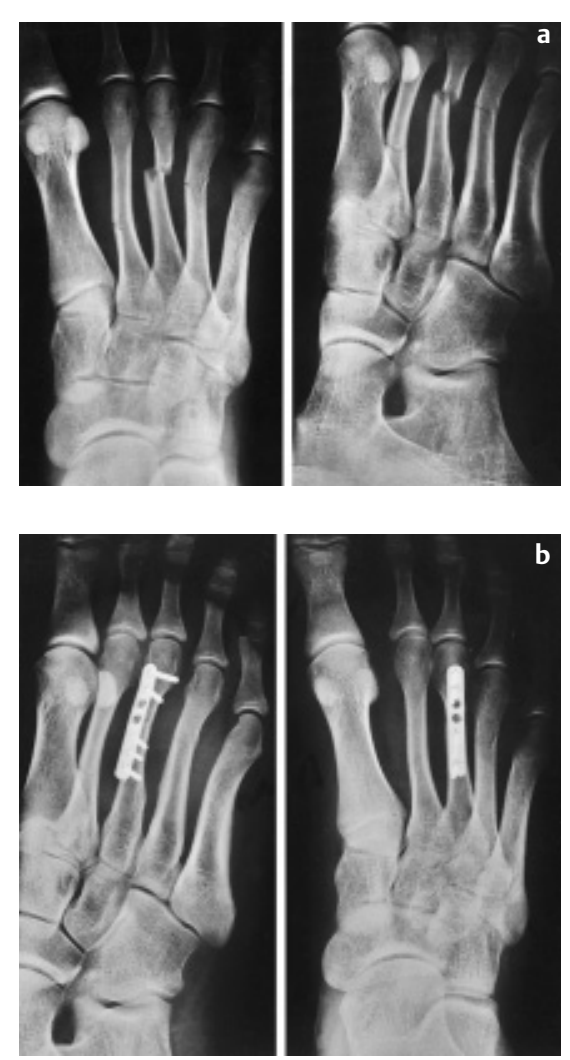

Abb.4 Dislozierte, diaphysäre Querfraktur des Metatarsale III, undislozierte Schaftfrakturen der Metatarsalia II und IV bei Sturz mit Snowboard. Versorgung des Metatarsale III mit gerader 2,0-mm-Titanplatte. (a) Unfallbild, (b) postoperatives Röntgenbild.

Dabei sind eigentliche Basisfrakturen von einer Fraktur im Übergang der Metaphyse zum proximalen Schaft (Jonesfraktur) zu unterscheiden.

Während die Basisfrakturen häufig mit konservativen Behandlungsmethoden ausheilen, können die eigentlichen Jones-Frakturen Probleme bei der Frakturheilung darstellen. Die wiederholten Adduktionsbewegungen (welche ihrerseits möglicherweise einer operativen Sanierung bedürfen) und eine heikle Gefäßversorgung [7] - die proximale Metaphyse wird durch eine einzige A. nutritia versorgt, welche im Übergang der Metaphyse zur proximalen Diaphyse an der medialen Kortikalis einsprosst - verlangen eine differenzierte Behandlung, welche sich deutlich von der Behandlung der einfachen Basisfraktur unterscheidet.

\section{Basisfraktur Metatarsale V}

Die Einteilung dieser Frakturen erfolgt zweckmäßig nach Holzach [3]. Beim Typ I liegt die Frakturebene parallel

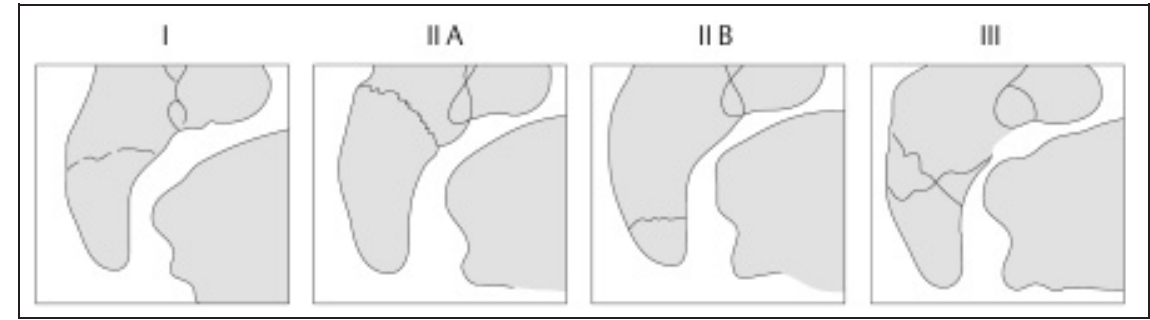

Abb. 5 Die Typeneinteilung der Basisfraktur des Metatarsale V nach Holzach.
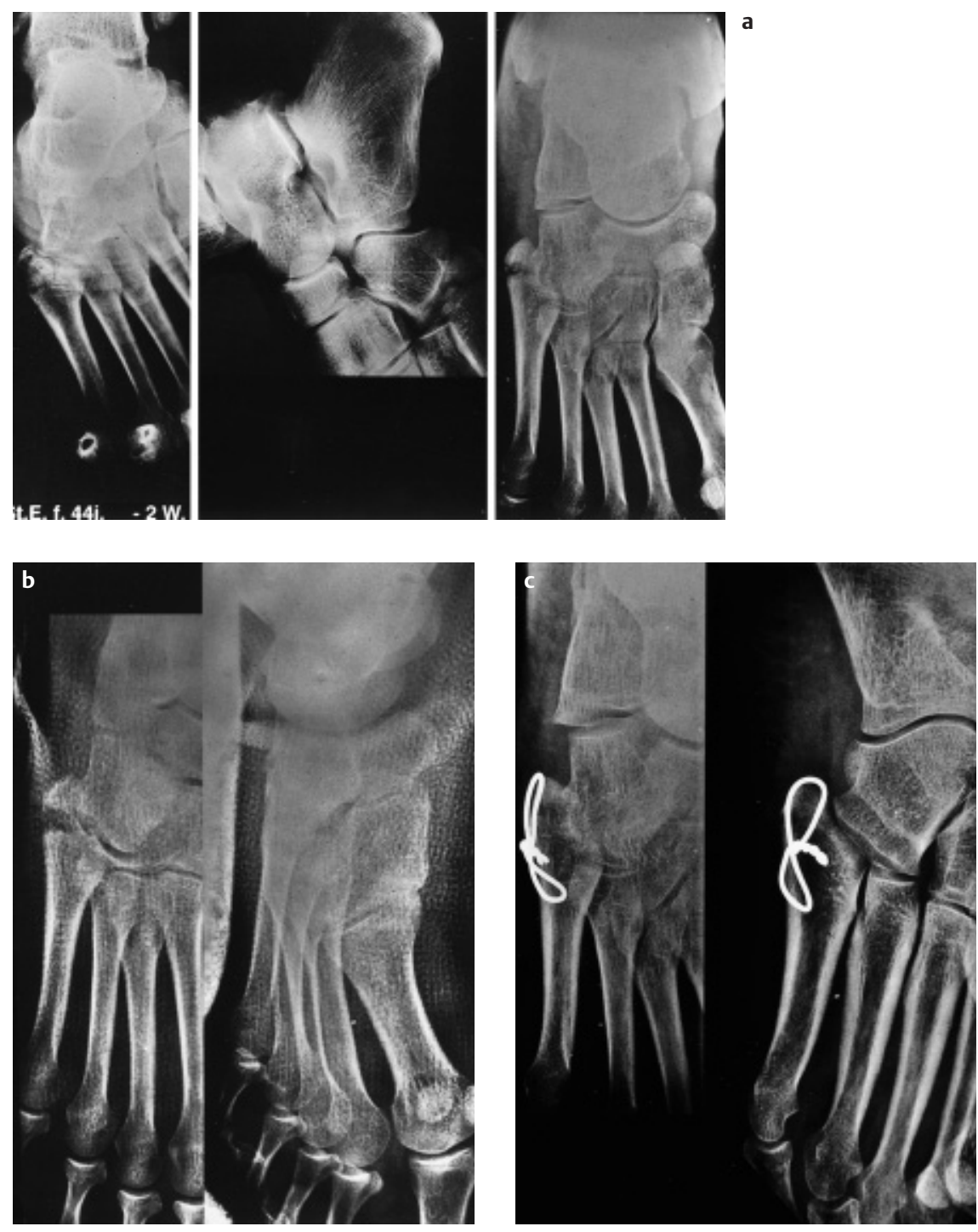

Abb. 6 Basisfraktur Typ II A des Metatarsale V bei Distorsionstrauma. Sekundär versorgt nach zunehmender Dislokation mit Drahtzuggurtung. (a) Unfallbild, (b) zunehmende Frakturdehiszenz nach 1 Woche, (c) postoperatives Röntgenbild. 

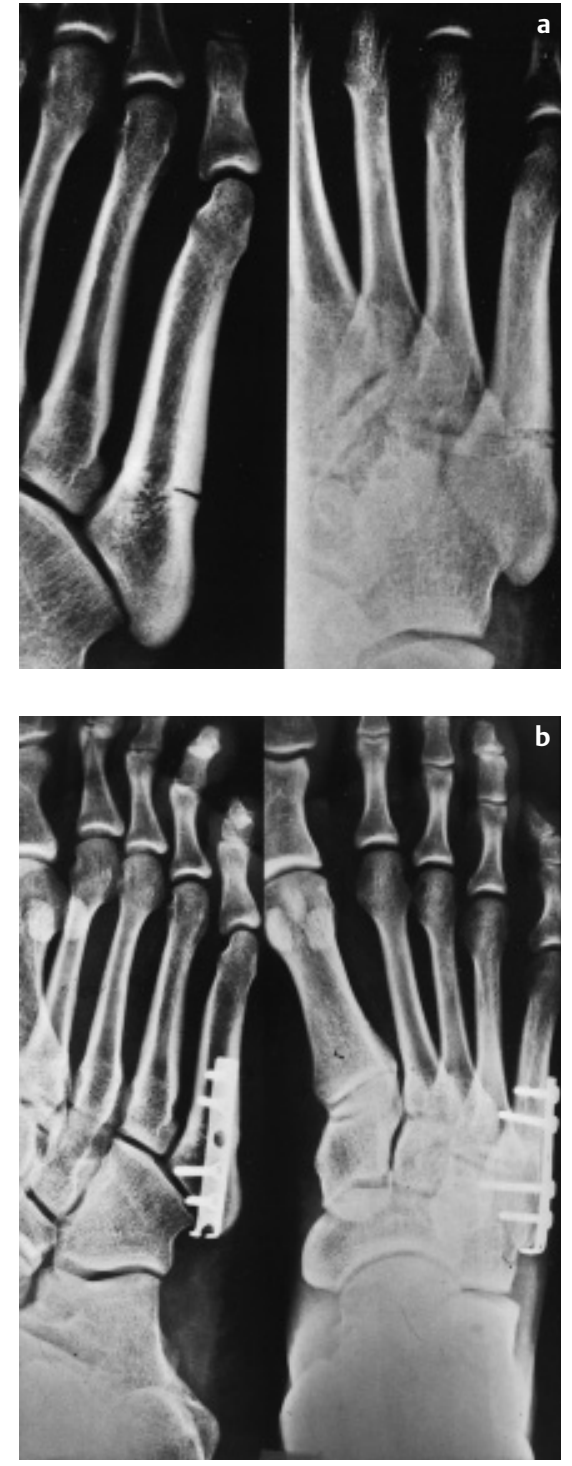

Abb.7 Jones-Fraktur Metatarsale V (Torg I) bei jungem Eishockeyspieler. Versorgt mit modifizierter Viertelrohrhakenplatte. (a) Unfallbild, (b) 1-Jahres-Röntgenbild.

zum tarsometatarsalen Gelenk. Beim Typ II liegt die Frakturebene quer zum Tarsometatarsalgelenk und verläuft teils extra-, teils intraartikulär. Typ III umfasst eine Kombination mehrerer Fragmente und Frakturebenen (Abb.5).

Typ-I-Frakturen können dank dem topographischen Zuggurtungsmechanismus problemlos konservativ behandelt werden. Gleiches gilt für die undislozierten Typ-II- und-III-Frakturen. Dabei scheinen die weniger rigiden Fixationen (Soft-cast oder Tapeverband) gegenüber den konventionellen Unterschenkelgipsen den Vorteil einer schnelleren Rehabilitation bei gleichbleibender Frakturheilungszeit

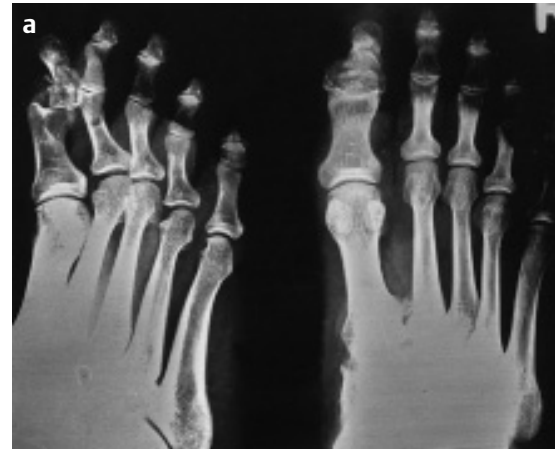

Tab. 1 Therapiekonzept der Basisfraktur des Metatarsale $\mathrm{V}$ unter Berücksichtigung der Einteilung nach Holzach [3]

\begin{tabular}{lll} 
Typ & Behandlung \\
\hline I & & Tape/Softcast \\
II & $\begin{array}{l}\text { undisloziert } \\
\text { disloziert }\end{array}$ & $\begin{array}{l}\text { Tape/Softcast } \\
\text { operativ }\end{array}$ \\
III & $\begin{array}{l}\text { undisloziert } \\
\text { disloziert }\end{array}$ & $\begin{array}{l}\text { Tape/Softcast } \\
\text { operativ }\end{array}$ \\
&
\end{tabular}

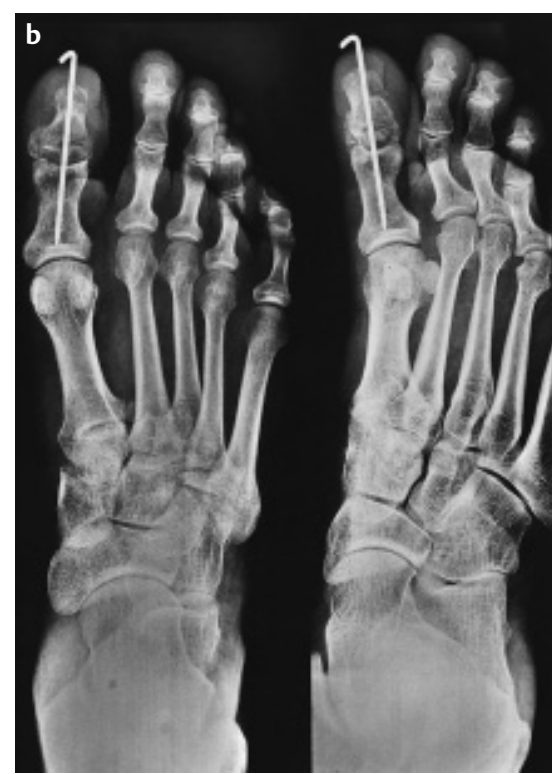

Abb. 8 Offene Trümmerfraktur der distalen Großzehen-Phalanx. Versorgt mit einer Kirschner-Draht-Osteosynthese mit temporärer IP-Arthrodese. (a) Unfallbild, (b) postoperatives Röntgenbild.

zu haben [10]. Bei zunehmender Frakturdiastase bei Typ-II- und -III-Frakturen jedoch empfiehlt sich eine innere Fixation (Tab.1) [5].

Operative Möglichkeiten der Basisfraktur des Metatarsale V sind die Zugschraubenosteosynthese oder eine Drahtzuggurtung mit/ohne zusätzliche Kirschnerdrähte (Abb.6). Bei perkutaner Schraubenosteosynthese ist die Möglichkeit einer Verletzung des N. suralis zu berücksichtigen [1]. Bei diesen übungsstabilen Osteosynthesen entfallen postoperative Ruhigstellungen in Gipsverband oder ähnlichen Verfahren.

\begin{tabular}{|c|c|c|}
\hline Typ & nach Torg & Behandlung \\
\hline I & akut & $\begin{array}{l}\text { konservativ } \\
\text { operativ (Sportler) }\end{array}$ \\
\hline II & $\begin{array}{l}\text { verzögerte } \\
\text { Heilung }\end{array}$ & operativ \\
\hline III & $\begin{array}{l}\text { Pseudoar- } \\
\text { throse }\end{array}$ & operativ \\
\hline
\end{tabular}

Jones-Fraktur

Anders und weniger einheitlich ist die Behandlung der sogenannten Jones-Fraktur, jener Fraktur, die sich im Übergang der Metaphyse zum proximalen Metatarsale-V-Schaft ereignet [4]. Aus Gründen der Vaskularität (Eintrittsstelle der einzigen A. nutritia genau auf Höhe der Fraktur) [7] und der frakturinduzierenden Faktoren (Adduktionskräfte) [9] zeigt dieser Frakturtyp eine Tendenz zur Pseudoarthrosenbildung.

Die Einteilung nach Torg [8] hat sich ähnlich der Einteilung der Basisfrakturen nach Holzach [3] - bewährt, da sie therapeutische Implikationen beinhaltet.

Typ I ist eine frische, akute Fraktur mit scharfen Frakturlinien ohne intramedulläre Sklerose. Typ II, eine Fraktur mit verzögerter Heilung, im Röntgenbild erkennbar durch Frakturspalterweiterung mit einer Frakturlinie in beiden Cortices mit periostaler Knochenneubildung und intramedullärer Sklerose. Der Typ III ist eine etablierte Pseudoarthrose mit völliger Obliteration der Markhöhle durch sklerotischen Knochen. Dieses Einteilungs- und Therapieschema ist in Tab. 2 wiedergegeben.

Operative Therapiemöglichkeiten der Jonesfraktur des Typ I beim Sportler, Typ II 
und III nach Torg [8] sind: Markhöhlendebridement und kortikospongiöser Span (Methode nach Torg), intramedulläre Zugschraubenosteosynthese (kanülierte Schraube) oder eine dorsolaterale Plattenosteosynthese (Viertelrohrplatte als modifizierte Hakenplatte) mit axialer Kompression (Abb. 7).

\section{Zehenfrakturen}

Die meisten Zehenfrakturen können konservativ zum Beispiel mit Dachziegelverbänden (Schienung des frakturierten Zehens mittels Klebverbänden an den Fußrücken, eventuell unter Einbezug des Nachbarzehens) behandelt werden. Frakturen der proximalen Phalanx des Großzehens sind häufig disloziert und unstabil wegen der Zugkräfte der Sehnen. Deshalb empfiehlt sich hier eine interne Fixation mittels Miniplatten (T-Platten oder Minikondylenplatten) [2]. Massiv dislozierte Phalanxfrakturen der anderen Zehen können oft mit einer perkutanen - oder bei offenen Frakturen unter Sicht - intramedullären Kirschner-Draht-Schienung fixiert werden, eventuell unter temporärer Arthrodese der benachbarten Gelenke (Abb.8). Bei dieser Methode besteht keine genügende Stabilität, weshalb diese Markdrahtschienung häufig mit einem Gehgips oder einem Halluxschuh mit harter Schuhsohle nachbehandelt werden muss.

In den letzten Jahren zeigten sich, sowohl im Behandlungsmanagement (konservativ oder operativ) als auch in der operativen Technik, einige neue Trends.

Unter dem Aspekt der anatomischen Wiederherstellung, welche mit dem funktionellen Behandlungsresultat in direktem Zusammenhang steht, hat sich ähnlich wie bei der distalen Radiusfraktur - ein gewisser Trend zur operativen Sanierung bei allen dislozierten Metatarsaleschaftfrakturen vollzogen.

Anstelle der einfacheren, intramedullären Kirschner-Draht-Schienung bei Metatarsaleschaftfrakturen II - IV ist ein Trend zur aufwendigeren, übungsstabilen Miniplattenosteosynthese festzustellen.

Neu wird neben den erwähnten Implantaten auch über eine Verwendung der sog. Herbert-Schraube (intramedulläre Kompressionsschraubenosteosynthese mit Schrauben-Doppelgewinde) bei Pseudoarthrosen und Stressfrakturen berichtet [6].

\section{Literatur}

${ }^{1}$ Donley BG, McCollum MJ, Murphy GA et al. Risk of sural nerve injury with intramedullary screw fixation of fifth metatarsal fractures: A cadaver study. Foot \& Ankle International 1999; 20: $182-184$

${ }^{2}$ Heim U, Pfeiffer KM. Periphere Osteosynthesen. Springer Verlag, Berlin, Heidelberg, New York, London, Paris, Tokyo 1988

${ }^{3}$ Holzach P, Staubli A, Gerber B. Die Behandlung der Basisfraktur des Os metatarsale. Helv Chir Acta 1983; 50: 69-72

${ }^{4}$ Jones R. Fracture of the base of the fifth metatarsal bone by indirect violence. Ann Surg 1902; 35: 697-700

${ }^{5}$ Linder P, Heim D, Braun R et al. Metatarsale V Basisfraktur. Z Unfallchir Vers med Berufskr 1986; 79: 257-260

${ }^{6}$ Sammarco GJ, Carrasquillo HA. Intramedullary fixation of metatarsal fracture and nonunion. Orthop Clin North Am 1995; 26 : 265-272

${ }^{7}$ Smith JW, Arnoczky SP, Hersh A. The intraosseous blood supply of the fifth metatarsal: Implications for proximal fracture healing. Foot \& Ankle 1992; 13: $143-152$

${ }^{8}$ Torg JS, Balduini FC, Zelko RR et al. Fractures of the base of the fifth metatarsal distal to the tuberosity. J Bone Joint Surg 1984; 66A: 209-214

${ }^{9}$ Weinfeld SB, Haddad SL, Myerson MS. Metatarsal stress fractures. Clin Sports Med 1997. 16: $319-338$

${ }^{10}$ Wiener BD, Linder JF, Giattini JFG. Treatment of fractures of the fifth metatarsal: A prospective study. Foot \& Ankle International 1997; 18: 267-269

Priv.-Doz. Dr. med. Dominik Heim Chefarzt Chirurgie

Dr. med. Ulrich Stricker

Co-Chefarzt Chirurgie

Spital Frutigen

Postfach

CH-3714 Frutigen

Schweiz 